\title{
STABILITY AND INSTABILITY OF POSITIVE SOLUTIONS OF SEMIPOSITONE PROBLEMS
}

\author{
ACHILLES TERTIKAS
}

(Communicated by Barbara L. Keyfitz)

Abstract. We consider the boundary value problem

$$
-\Delta u=f(u) \text { in } \Omega, \quad B u=0 \text { on } \partial \Omega,
$$

where $\Omega$ is a bounded region in $\mathbb{R}^{n}$ with smooth boundary. We prove stability and instability results of positive solutions under various choices of $f$.

\section{INTRODUCTION}

In this note we consider the stability of positive solutions of the semilinear elliptic problem

$$
\begin{gathered}
-\Delta u(x)=f(u(x)), \quad x \in \Omega, \\
B u(x)=0, \quad x \in \partial \Omega,
\end{gathered}
$$

where $\Omega$ is a bounded domain in $\mathbb{R}^{n}$ having smooth boundary, $\Delta$ is the Laplace operator, and $B$ is defined by

$$
B u(x)=a h(x) u(x)+(1-a) \frac{\partial u(x)}{\partial n}
$$

with $a \in[0,1], h: \partial \Omega \rightarrow \mathbb{R}^{+}$is a smooth function with $h \equiv 1$ when $a=1$. We call a function $u$ positive if $u \geq 0$ and $u \neq \equiv$ in $\bar{\Omega}$.

Stability of solutions is described through the initial value problem

$$
\begin{gathered}
u_{t}=\Delta u+f(u), \quad x \in \Omega, t>0, \\
B u=0, \quad x \in \partial \Omega, t>0, \\
u(x, 0)=u_{0}(x), \quad x \in \Omega .
\end{gathered}
$$

In this way a solution $U$ of (1.1), (1.2) is called $L^{\infty}$ stable if for any $\varepsilon>0$ there exists $\delta>0$ such that when $u_{0}$ satisfies $\left\|u_{0}-U\right\|_{\infty}<\delta$ then

$$
\|u(\cdot, t)-U\|_{\infty}<\varepsilon \quad \text { for all } t \geq 0 .
$$

Received by the editors October 23, 1990.

1980 Mathematics Subject Classification (1985 Revision). Primary 35B35, $35 \mathrm{~J} 65$.

Key words and phrases. Subsupersolutions, linearized stability, positone. 
A solution is called $L^{\infty}$ unstable when it is not $L^{\infty}$ stable. Very recently Brown and Shivaji [1] proved instability of positive solutions of (1.1), (1.2) when $f$ is a smooth function satisfying

$$
\begin{gathered}
f(0)<0, \\
f^{\prime}(u)>0, \text { for } u>0, \\
f^{\prime \prime}(u) \geq 0, \text { for } u>0 .
\end{gathered}
$$

We aim to improve their result in the best possible way. To do this and to avoid the problem being linear we set

$$
\begin{array}{ll}
f(0)=0 \text { and } f^{\prime \prime}(u)>0 & \text { for } u>0 \quad \text { or } \\
f(0)<0 \text { and } f^{\prime \prime}(u) \geq 0 & \text { for } u>0
\end{array}
$$

and prove

Theorem 1. Suppose $f$ satisfies (F). Then every positive solution of (1.1), (1.2) is $L^{\infty}$ unstable.

We consider the problem

$$
\begin{gathered}
\Delta u+\lambda e^{u}=0, \quad \text { in } B(0,1), \\
u=0, \text { on } \partial B(0,1),
\end{gathered}
$$

which has been studied by Fujita [2] and Joseph and Lundgren [4]. Take $1 \leq$ $n \leq 9$, then (1.11), (1.12) might have multiple positive solutions, the smallest of which is stable and any other unstable. This suggest that no general statement concerning the stability or instability of all solutions can be made when $f^{\prime \prime}>0$ and $f(0)>0$.

However if we replace convexity of $f$ by concavity, that is

$$
\begin{array}{ll}
f(0)=0 \text { and } f^{\prime \prime}(u)<0 & \text { for } u>0 \quad \text { or } \\
f(0)>0 \text { and } f^{\prime \prime}(u) \leq 0 & \text { for } u>0,
\end{array}
$$

then

Theorem 2. Suppose $f$ satisfies $(\mathrm{G})$. Then very positive solution of (1.1), (1.2) is $L^{\infty}$ stable. Moreover there is at most one such solution.

Remark 1. Theorem 2 is well known.

Remark 2. Our method extends to more general elliptic operators, as well as to more general nonlinearities, but we will not labour this point any further.

\section{Proofs}

Initially we prove

Lemma 1. (a) Suppose $f$ satisfies (F). Then

$$
u f^{\prime}(u)-f(u)>0 \quad \text { for } u>0 .
$$

Also

$$
-t f(u)+f(t u)>0 \quad \text { for } t>1, u>0,
$$

and

$$
-t f(u)+f(t u)<0 \quad \text { for } 0<t<1, u>0 .
$$


(b) Suppose $f$ satisfies $(\mathrm{G})$. Then

$$
u f^{\prime}(u)-f(u)<0 \text { for } u>0 \text {. }
$$

Also

$$
-t f(u)+f(t u)<0 \quad \text { for } t>1, u>0
$$

and

$$
-t f(u)+f(t u)>0 \quad \text { for } 0<t<1, u>0 .
$$

Proof. The proof is quite elementary and we will give it only in the first case. Hence we suppose that $f$ is such that $f(0)<0$ and $f^{\prime \prime}(u) \geq 0$ for $u>0$.

Let $g(u)=u f^{\prime}(u)-f(u)$. Then

$$
g^{\prime}(u)=u f^{\prime \prime}(u) \geq 0 \quad \text { for } u>0 .
$$

Hence

$$
g(u) \geq g(0)=-f(0)>0 \text { for } u>0
$$

Therefore

$$
u f^{\prime}(u)-f(u)>0 \text { for } u>0 \text {. }
$$

Now consider the function $h$ defined by

$$
h(t)=-f(u)+\frac{f(t u)}{t} \quad \text { for } t>0, u>0 .
$$

It is easy to see that

$$
h^{\prime}(t)=\frac{t u f^{\prime}(t u)-f(t u)}{t^{2}}>0 \text { for } t>0, u>0
$$

and $h(1)=0$. These suggest that

$$
h(t)>h(1)=0 \quad \text { for } t>1, u>0
$$

or

$$
-t f(u)+f(t u)>0 \text { for } t>1, u>0
$$

and

$$
h(t)<0 \quad \text { when } 0<t<1, u>0
$$

which gives

$$
-t f(u)+f(t u)<0 \quad \text { when } 0<t<1, u>0 .
$$

When $f(0) \neq 0$ we can easily see that Lemma 1 is valid for all $u \geq 0$. Also, when $f(0)=0$, by applying the maximum principle, we can conclude the strict positivity of our solutions for all $x \in \Omega$. We therefore may apply Lemma 1 for our positive solutions.

Suppose now $u$ is a positive solution of (1.1), (1.2). The linearized equation associated with (1.1), (1.2) is

$$
\begin{gathered}
-\Delta \phi-f^{\prime}(u) \phi=\lambda \phi \quad \text { in } \Omega, \\
B \phi=0 \quad \text { on } \partial \Omega .
\end{gathered}
$$

Let $\lambda_{1}$ be the smallest eigenvalue and $\phi_{1}$ be the corresponding eigenfunction. We may take $\phi_{1}$ such that $\phi_{1}(x)>0$ in $\Omega$. 
Lemma 2. (a) Suppose $f$ satisfies (F). Then $\lambda_{1} \leq 0$.

(b) Suppose $f$ satisfies $(\mathrm{G})$. Then $\lambda_{1} \geq 0$.

Proof. (a) We set

$$
u_{\varepsilon}(x)=\varepsilon u(x) \quad \text { for } \varepsilon>0 \text { and } x \in \Omega \text {. }
$$

Then we have

$$
\Delta u_{\varepsilon}(x)+f\left(u_{\varepsilon}(x)\right)=-\varepsilon f(u(x))+f(\varepsilon u(x)), \quad x \in \Omega .
$$

Hence by Lemma 1 we conclude that

$$
\Delta u_{\varepsilon}(x)+f\left(u_{\varepsilon}(x)\right)>0 \quad \text { if } \varepsilon>1, x \in \Omega
$$

and

$$
\Delta u_{\varepsilon}(x)+f\left(u_{\varepsilon}(x)\right)<0 \quad \text { if } 0<\varepsilon<1, x \in \Omega .
$$

At the same time it is easy to see that

$$
B u_{\varepsilon}(x)=0, \quad x \in \partial \Omega
$$

and that $u_{\varepsilon} \geq u$ or $u_{\varepsilon} \leq u$ depending on wheither $\varepsilon>1$ or $0<\varepsilon<1$ respectively.

This suggests that $u_{\varepsilon}$ is a strict subsolution of $\varepsilon>1$ and a strict supersolution when $0<\varepsilon<1$. Therefore by the principle of linearized stability [3] we conclude $\lambda_{1} \leq 0$.

(b) Similarly we set

$$
u_{\varepsilon}(x)=\varepsilon u(x) \quad \text { for } \varepsilon>0, x \in \Omega .
$$

Then

$$
\Delta u_{\varepsilon}(x)+f\left(u_{\varepsilon}(x)\right)<0 \quad \text { if } \varepsilon>1, x \in \Omega
$$

and

$$
\Delta u_{\varepsilon}(x)+f\left(u_{\varepsilon}(x)\right)>0 \quad \text { if } 0<\varepsilon<1, x \in \Omega .
$$

Thus if $0<\varepsilon<1, u_{\varepsilon}(x) \leq u(x), x \in \Omega$ is a strict subsolution, and if $\varepsilon>1$, $u_{\varepsilon}(x) \geq u(x), x \in \Omega$ is a strict supersolution. The result follows.

Proof of Theorem 1. By Lemma 2 (a), we have $\lambda_{1} \leq 0$. The result will follow by the principle of linearized stability [3] once we prove that $\lambda_{1}<0$. Suppose the contrary that $\lambda_{1}=0$.

We multiply (1.13) by $u$ and integrate to obtain

$$
-\int_{\Omega} u \Delta \phi_{1} d x-\int_{\Omega} f^{\prime}(u) u \phi_{1} d x=0
$$

or

$$
\int_{\Omega} \nabla u \nabla \phi_{1} d x-\int_{\partial \Omega} u \frac{\partial \phi_{1}}{\partial n} d s-\int_{\Omega} f^{\prime}(u) u \phi_{1} d x=0 .
$$

Similarly multiplying (1.1) by $\phi_{1}$ and integrating we finally conclude

$$
\int_{\Omega} \nabla \phi_{1} \nabla u d x-\int_{\partial \Omega} \phi_{1} \frac{\partial u}{\partial n} d s-\int_{\Omega} f(u) \phi_{1} d x=0 .
$$

Subtracting (1.15), (1.16) we arrive at

$$
\int_{\partial \Omega}\left\{\phi_{1} \frac{\partial u}{\partial n}-u \frac{\partial \phi_{1}}{\partial n}\right\} d s+\int_{\Omega}\left\{f(u)-u f^{\prime}(u)\right\} \phi_{1} d x=0 .
$$


However

$$
\int_{\partial \Omega}\left\{\phi_{1} \frac{\partial u}{\partial n}-u \frac{\partial \phi_{1}}{\partial n} d s\right\}=0
$$

since it is obvious when $a=1$ and when $a \neq 1$, then

$$
\phi_{1} B u-u B \phi_{1}=(1-a)\left\{\phi_{1} \frac{\partial u}{\partial n}-u \frac{\partial \phi_{1}}{\partial n}\right\} \text {. }
$$

Therefore (1.17) gives

$$
\int_{\Omega}\left\{f(u)-u f^{\prime}(u)\right\} \phi_{1} d x=0
$$

and by Lemma 1 we can easily arrive at a contradiction.

Proof of Theorem 2. As in the proof of Theorem 1 we can easily obtain that $\lambda_{1} \neq 0$. Hence by Lemma 2 what remains to be proved is the uniqueness of positive solutions.

Suppose on the contrary $u, v$ are two distinct solutions with

$$
\max _{x \in \mathbf{\Omega}}\{u(x)-v(x)\}>0
$$

and set $u_{\varepsilon}(x)=\varepsilon u(x), x \in \Omega$.

By taking $\varepsilon$ large enough we may suppose that

$$
u_{\varepsilon}(x) \geq v(x) \quad x \in \Omega\left(u_{\varepsilon} \not \equiv v\right)
$$

and by Lemma 1 , one can easily conclude that $u_{\varepsilon}$ is a strict supersolution. Therefore we may suppose that (1.1), (1.2) have a positive solution $w$ such that

$$
w(x) \geq \max (u(x), v(x)), \quad x \in \Omega
$$

and because of (1.19), (1.20) and the strong maximum principle that

$$
w(x)>v(x), \quad x \in \Omega
$$

by multiplying (1.1) by $w$ and $v$ and subtracting, we can very easily conclude

$$
\int_{\partial \Omega}\left\{u \frac{\partial w}{\partial n}-w \frac{\partial v}{\partial n}\right\} d s+\int_{\Omega} w v\left\{\frac{f(w)}{w}-\frac{f(v)}{v}\right\} d x=0
$$

and using the boundary conditions that

$$
\int_{\Omega} w v\left\{\frac{f(w)}{w}-\frac{f(v)}{v}\right\} d x=0
$$

The contradiction comes using (1.21) and

$$
\left(\frac{f(u)}{u}\right)^{\prime}=\frac{u f^{\prime}(u)-f(u)}{u^{2}}<0 \text { for } u>0 .
$$

\section{ACKNOWLEDGMENT}

The author wishes to thank Dr. K. J. Brown for providing [1] prior to publication and for encouraging the author to write this note. 


\section{REFERENCES}

1. K. J. Brown and R. Shivaji, Instability of nonnegative solutions for a class of semipositone problems, Proc. Amer. Math. Soc. 112 (1991), 121-124.

2. H. Fujita, On the nonlinear system equations $\Delta u+e^{u}=0$ and $v_{t}=\Delta v+e^{v}$, Bull. Amer. Math. Soc. 75 (1969), 132-135.

3. D. Henry, Geometric theory of semilinear parabolic equations, Lecture Notes in Math., vol. 840, Springer-Verlag, Berlin and New York, 1981.

4. D. D. Joseph and T. S. Lundgren, Quasilinear Dirichlet problems driven by positive sources, Arch. Rational Mech. Anal. 49 (1973), 241-269.

Department of Mathematics, University of Crete, P. O. Box 1470, Iraklion, Crete, GREECE 